\title{
Dairy Business Analysis Project: 2007 Financial Summary 1
}

A. De Vries, R. Giesy, M. Sowerby, and L. Ely ${ }^{2}$

\section{Introduction}

The Dairy Business Analysis Project (DBAP) was initiated in 1996 by the University of Florida in an effort to measure and document the financial performance of Florida dairy farms using standardized accounting measures. The University of Georgia has been a formal collaborator since 1998. The DBAP Web site is http://dairy.ifas.ufl.edu/dbap.

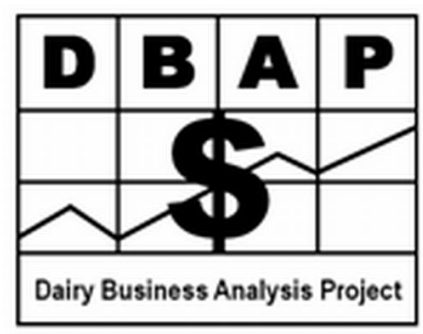

Financial data for the year 2007 were collected from 21 participating dairy farms and screened for completeness and validity. Each dairy farm then received a benchmark report detailing its financial results compared to the average results for the other participants and the six dairy farms with the highest net farm income per cwt. This benchmark report is discussed with the participants to identify challenges and opportunities for improvement.
This publication is a summary of the financial performance of the 21 dairy farms that participated in 2007. It is intended for general use by dairy farmers, the allied industry, government, and educational professionals.

\section{Data Collection and Accounting Methods}

Dairy producers in Florida and Georgia were given the opportunity to participate in DBAP. Participants were not a random sample of all dairy farms in the two states. The financial performance results in this publication are therefore not necessarily representative of the average results of all dairy farms in Florida and Georgia.

Most of the data were collected by extension agents when visiting dairy farms using a standardized data collection spreadsheet. Occasionally, data were sent in by the dairy producers. The financial data were either entered into the spreadsheet on the farm or mailed in on paper copies of the spreadsheet.

The accounting methods followed the recommendations made by the Farm Financial

1. This document is AN213, one of a series of the Animal Science Department, Florida Cooperative Extension Service, Institute of Food and Agricultural Sciences, University of Florida. Original publication date March 2009. Visit the EDIS Web Site at http://edis.ifas.ufl.edu.

2. A. De Vries, Associate Professor, Department of Animal Sciences; R. Giesy, Extension Agent IV (retired); M. Sowerby, Extension Agent III; L. Ely, Professor, Department of Animal and Dairy Science, University of Georgia;Cooperative Extension Service, Institute of Food and Agricultural Sciences, University of Florida, Gainesville, 32611.

The Institute of Food and Agricultural Sciences (IFAS) is an Equal Opportunity Institution authorized to provide research, educational information and other services only to individuals and institutions that function with non-discrimination with respect to race, creed, color, religion, age, disability, sex, sexual orientation, marital status, national origin, political opinions or affiliations. U.S. Department of Agriculture, Cooperative Extension Service, University of Florida, IFAS, Florida A. \& M. University Cooperative Extension Program, and Boards of County Commissioners Cooperating. Larry Arrington, Dean 
Standards Council. All revenues and expenses were accrual adjusted. Cash receipts and expenses were therefore adjusted for changes in inventory, prepaid expenses, accounts payable, and accounts receivable. Depreciation data were often taken from tax records. Asset valuation was based on market values if available, but the changes from January 1 to December 31 were kept small. Unpaid management was valued at $\$ 50,000$ per farm. Gain or loss on sale of purchased livestock resulted when livestock depreciation did not completely account for the gain or loss in the value of the purchased livestock during 2007. Appreciation resulted when machinery and building depreciation did not completely account for the gain or loss in the value of these capital assets during 2007. The bottom line of each dairy farm is its net farm income. Net farm income is the return to the owner and unpaid family members for their labor, management, and equity in the dairy farm. It is the total income available for owner's salary, new investments, taxes, and paying off principal.

All submitted data were carefully scrutinized and checked for completeness. The cash flow statement reconciles the net cash flow resulting from the reported operating, investing, and financing activities with the reported available cash on the January 1 and December 31 balance sheets. The equity statement reconciles the changes in equity through reported retained capital and valuation with the calculated equity on the balance sheets. The reconciliation attempts typically result in unresolved imbalances. Both cash flow imbalance and equity imbalance had to be less than $10 \%$ to be included in the summary results reported here.

All results in this publication are the simple averages of the statistics of the dairy farms with valid data. Every dairy farm has equal weight. For example, assume a 100-cow herd produced 1,700,000 lbs of milk (17,000 lbs / cow) and a 1000-cow herd produced 19,000,000 lbs of milk (19,000 lbs / cow). Average milk yield per farm is $(19,000,000+$ $1,700,000) / 2=10,350,000 \mathrm{lbs}$ and average herd size is $(100+1000) / 2=550$ cows. The weighted average milk yield per cow is $10,350,000 / 550=$ $18,818 \mathrm{lbs} / \mathrm{cow}$. The simple average milk yield per cow is $(17,000+19,000) / 2=18,000 \mathrm{lbs} /$ cow .
Some definitions and calculation rules are as follows:

Asset turnover ratio $=$ total revenues $/$ average assets

Assets $=$ value of assets on the balance sheet

Average assets $=$ average of value of assets on January 1 and December 31

Average equity $=$ average of value of equity on January 1 and December 31

Capital replacement and term debt repayment margin $=\mathrm{NFIFO}+$ depreciation + interest on term debt - net social security and income taxes - owner withdrawals - annual scheduled payments on term debt and capital leases

Cash flow coverage ratio $=($ cash revenues - cash expenses) / current liabilities

Current assets $=$ short-term assets that can be utilized within one year

Current liabilities $=$ liabilities due within one year

Current ratio $=$ current assets $/$ current liabilities

Debt to asset ratio $=$ liabilities $/$ assets

Debt to equity ratio $=$ liabilities $/$ equity

Depreciation expense ratio $=$ depreciation $/$ total revenue

Equity $=$ assets - liabilities

Equity to asset ratio $=$ equity $/$ assets

FTE $=$ full time equivalent worker, on average 54 hours per week

Interest expense ratio $=$ interest paid $/$ total revenue

Liabilities $=$ value of liabilities on the balance sheet

Net farm income $=$ NFIFO + gain on sale of capital assets

$\mathrm{NFIFO}=$ net farm income from operations 
NFIFO ratio $=$ NFIFO $/$ total revenue

Operating expense ratio $=($ total operating expenses - depreciation) / total revenue

Operating profit margin ratio $=(\mathrm{NFIFO}+$ interest paid - unpaid management) / total revenues

Rate of return on dairy assets $=(\mathrm{NFIFO}+$ interest paid - unpaid management) / average assets

Rate of return on equity $=(\mathrm{NFIFO}-$ unpaid management) / average equity

Std $=$ standard deviation

Term debt and capital lease coverage ratio $=($ NFIFO + non-dairy income + depreciation + interest paid on term debt - net social security and income taxes owner withdrawals) / (annual scheduled payments on term debt and capital leases).

Working capital $=$ current assets - current liabilities

\section{Results}

Summary results for 2007 are presented in Tables 1.1 to 4.4 and Figures 1 to 5. Each category in Tables 2.1 to 4.4 is divided into three levels based on the lowest 7, middle 7, and highest 7 farms for that category. Therefore, the lowest 7 farms in one category may be different from the lowest 7 farms in another category. Revenues and expenses may not add up due to rounding.

In brief, 21 dairy farms were included in the summary results. Of these, 16 were located in Florida and 5 in Georgia. The average herd size of the participating dairies was 1399 cows and 708 heifers with 18,410 lbs. milk sold per cow. The average culling rate was $35 \%$. The average milk price was $\$ 22.59$. Average total revenues were $\$ 14.67$ per cwt. milk sold. Total expenses averaged $\$ 21.09$ per cwt. sold. The largest items were purchased feed, $\$ 9.20$, and personnel costs, $\$ 3.57$ per cwt. sold. Net farm income from operations averaged $\$ 3.58$ per cwt. sold. Net farm income per cwt. was $\$ 3.57$. These results indicate a marked increase in average profitability compared to 2006 .

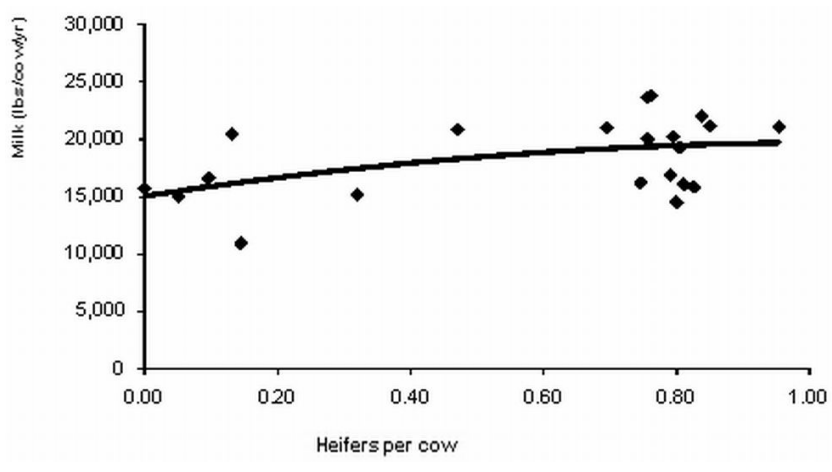

Figure 1. DBAP 2007 Summary - Milk production (lbs / cow / year) by heifers per cow

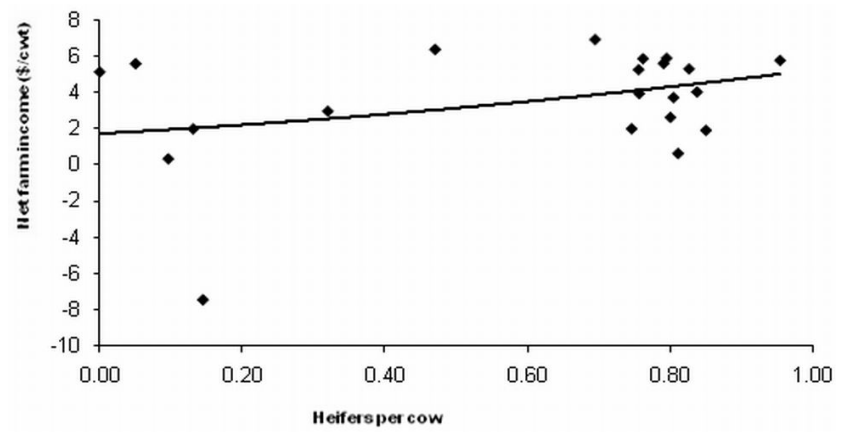

Figure 2. DBAP 2007 Summary - Net farm income (\$ / cwt) by heifers per cow

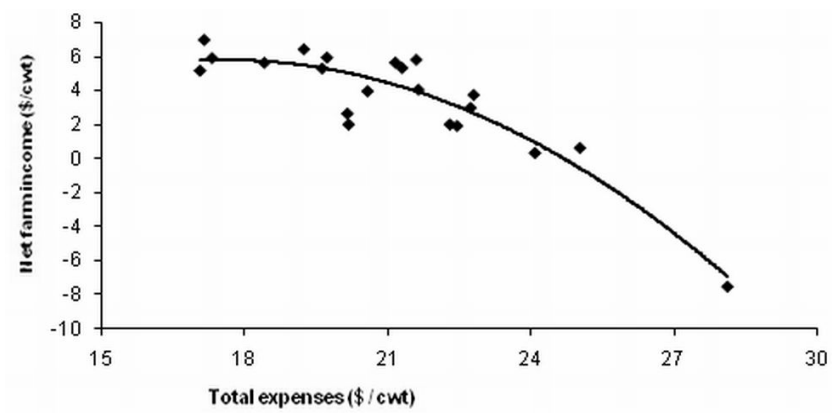

Figure 3. DBAP 2007 Summary - Net farm income (\$ / cwt) by total expenses ( $\$$ / cwt)

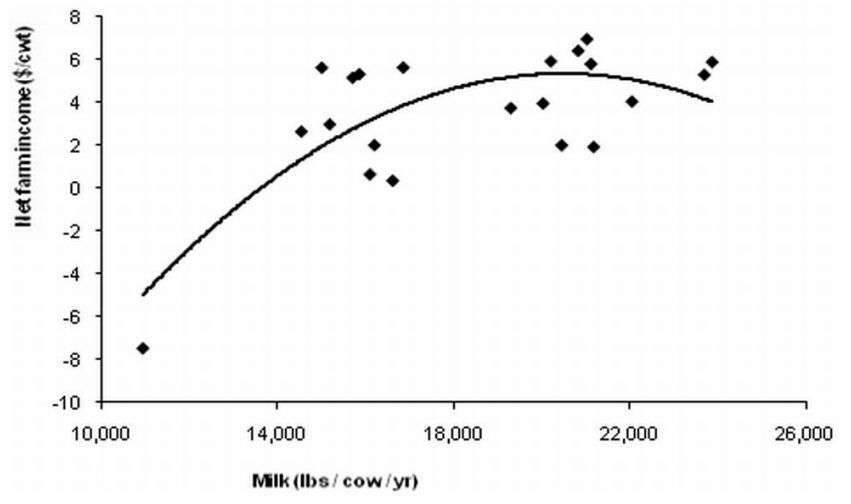

Figure 4. DBAP 2007 Summary - Net farm income ( $\$$ / cwt) by milk yield (lbs / cow / year) 


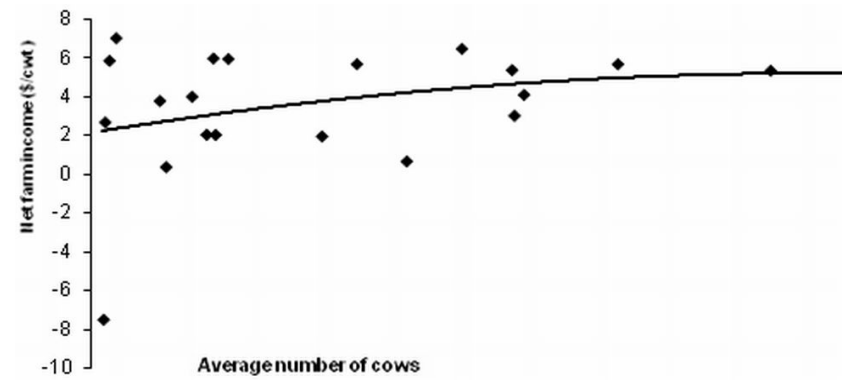

Figure 5. DBAP 2007 Summary - Net farm income per cwt (\$) by average number of cows. The x-axis is not displayed to avoid possible identification of dairy farms

Table 1.1. DBAP 2007 Summary - Business size and production efficiency by state and overall average, median, and standard deviation.

\begin{tabular}{|c|c|c|c|c|c|}
\hline \multirow[b]{2}{*}{ Category } & \multicolumn{3}{|c|}{ Overall } & \multicolumn{2}{|c|}{ State Averages } \\
\hline & Average & Median & $\operatorname{Std}^{1}$ & Florida & Georgia \\
\hline Number of farms & 21 & 21 & 21 & 16 & 5 \\
\hline \multicolumn{6}{|l|}{ Business Size: } \\
\hline Number of cows & 1,399 & 755 & 1,298 & 1,601 & 751 \\
\hline Number of heifers & 708 & 475 & 772 & 738 & 613 \\
\hline Milk sold (million lbs) & 25.73 & 18.01 & 24.07 & 28.59 & 16.57 \\
\hline $\mathrm{FTE}^{2}$ workers & 22 & 15 & 20 & 24 & 17 \\
\hline Acres of pasture + cultivated land & 781 & 431 & 691 & 869 & 500 \\
\hline \multicolumn{6}{|l|}{ Production Efficiency: } \\
\hline Milk sold (Ibs / cow / year) & 18,410 & 19,290 & 3,403 & 17,455 & 21,464 \\
\hline Cows / FTE worker & 59 & 50 & 30 & 65 & 39 \\
\hline Milk sold (million Ibs)/ FTE worker & 1.06 & 0.96 & 0.49 & 1.12 & 0.84 \\
\hline Cull rate & $35 \%$ & $34 \%$ & $12 \%$ & $36 \%$ & $33 \%$ \\
\hline
\end{tabular}

\footnotetext{
${ }^{1}$ Standard deviation

${ }^{2}$ Full-time equivalent
} 
Table 1.2. DBAP 2007 Summary - Revenues and expenses by state and overall average, median, and standard deviation $(\$ / c w t)$.

\begin{tabular}{|c|c|c|c|c|c|}
\hline \multirow[b]{2}{*}{ Category } & \multicolumn{3}{|c|}{ Overall } & \multicolumn{2}{|c|}{ State Averages } \\
\hline & Average & Median & $\mathrm{Std}^{1}$ & Florida & Georgia \\
\hline Number of farms & 21 & 21 & 21 & 16 & 5 \\
\hline \multicolumn{6}{|l|}{ Revenues: } \\
\hline Milk sold & 22.59 & 22.40 & 1.27 & 22.77 & 22.03 \\
\hline Raised, leased cow sales & 1.18 & 0.70 & 1.80 & 1.25 & 0.98 \\
\hline Heifer sales & 0.18 & 0.27 & 1.55 & 0.01 & 0.73 \\
\hline $\begin{array}{l}\text { Gain on purchased livestock } \\
\text { Sales }\end{array}$ & $(0.27)$ & $(0.07)$ & 0.55 & $(0.32)$ & $(0.10)$ \\
\hline Other revenues & 0.98 & 1.08 & 1.49 & 0.73 & 1.79 \\
\hline Total revenues & 24.67 & 24.55 & 1.75 & 24.43 & 25.42 \\
\hline \multicolumn{6}{|l|}{ Expenses: } \\
\hline Personnel & 3.57 & 3.45 & 1.57 & 3.51 & 3.77 \\
\hline Purchased feed & 9.20 & 9.31 & 2.00 & 9.56 & 8.03 \\
\hline Crops & 0.59 & 0.45 & 0.62 & 0.61 & 0.52 \\
\hline Machinery & 1.13 & 0.93 & 0.63 & 1.19 & 0.94 \\
\hline Livestock & 1.62 & 1.54 & 0.66 & 1.61 & 1.64 \\
\hline Milk marketing & 1.14 & 1.18 & 0.37 & 1.04 & 1.44 \\
\hline Buildings and land & 0.60 & 0.53 & 0.51 & 0.65 & 0.46 \\
\hline Interest & 0.62 & 0.62 & 0.54 & 0.53 & 0.92 \\
\hline \multicolumn{6}{|l|}{ Depreciation: } \\
\hline Livestock & 0.84 & 0.46 & 0.84 & 0.90 & 0.67 \\
\hline Machinery & 0.45 & 0.41 & 0.32 & 0.43 & 0.52 \\
\hline Buildings & 0.36 & 0.31 & 0.31 & 0.36 & 0.37 \\
\hline Other expenses & 0.97 & 0.93 & 0.41 & 1.01 & 0.82 \\
\hline Total expenses & 21.09 & 21.17 & 2.70 & 21.40 & 20.11 \\
\hline Net farm income from operations & 3.58 & 4.06 & 3.22 & 3.04 & 5.31 \\
\hline Gain on sale of capital assets & $(0.01)$ & 0.00 & 0.43 & $(0.01)$ & $(0.02)$ \\
\hline Net farm income & 3.57 & 4.06 & 3.19 & 3.03 & 5.29 \\
\hline
\end{tabular}

\footnotetext{
${ }^{1}$ Standard deviation
} 
Table 1.3. DBAP 2007 Summary - Financial performance by state and overall average, median, and standard deviation.

\begin{tabular}{|c|c|c|c|c|c|}
\hline \multirow[b]{2}{*}{ Category } & \multicolumn{3}{|c|}{ Overall } & \multicolumn{2}{|c|}{ State Averages } \\
\hline & Average & Median & $\operatorname{Std}^{1}$ & Florida & Georgia \\
\hline Number of farms & 21 & 21 & 21 & 16 & 5 \\
\hline \multicolumn{6}{|l|}{ Liquidity: } \\
\hline Current ratio & 14.16 & 0.98 & 43.80 & 2.02 & 52.98 \\
\hline Working capital (\$) & 369,244 & 110,056 & 866,026 & 290,001 & 622,821 \\
\hline \multicolumn{6}{|l|}{ Solvency: } \\
\hline Debt to asset ratio & 0.29 & 0.26 & 0.21 & 0.27 & 0.34 \\
\hline Equity to asset ratio & 0.71 & 0.74 & 0.21 & 0.73 & 0.66 \\
\hline Debt to equity ratio & 0.60 & 0.35 & 0.70 & 0.55 & 0.74 \\
\hline \multicolumn{6}{|l|}{ Profitability: } \\
\hline Rate of return on assets & 0.15 & 0.17 & 0.18 & 0.16 & 0.14 \\
\hline Rate of return on equity & 0.26 & 0.19 & 0.51 & 0.27 & 0.22 \\
\hline Operating profit margin ratio & 0.13 & 0.16 & 0.20 & 0.10 & 0.21 \\
\hline \multicolumn{6}{|l|}{ Financial efficiency: } \\
\hline Asset turnover rate & 0.86 & 0.81 & 0.57 & 0.93 & 0.65 \\
\hline Operating expense ratio & 0.77 & 0.76 & 0.14 & 0.79 & 0.69 \\
\hline Depreciation expense ratio & 0.07 & 0.07 & 0.04 & 0.07 & 0.06 \\
\hline Interest expense ratio & 0.03 & 0.02 & 0.02 & 0.02 & 0.04 \\
\hline NFIFO ratio ${ }^{2}$ & 0.14 & 0.16 & 0.14 & 0.12 & 0.21 \\
\hline \multicolumn{6}{|l|}{ Repayment capacity: } \\
\hline Cash flow coverage ratio & 20.18 & 0.86 & 55.72 & 4.63 & 69.94 \\
\hline Term debt coverage ratio $^{3}$ & 3.63 & 1.48 & 4.71 & 4.42 & 1.09 \\
\hline Capital replacement margin ${ }^{4}(\$)$ & 704,737 & 569,504 & 877,533 & 840,219 & 271,194 \\
\hline
\end{tabular}

${ }^{1}$ Standard deviation

${ }^{2}$ Net farm income from operations ratio

${ }^{3}$ Term debt and capital lease coverage ratio

${ }^{4}$ Capital replacement and term debt repayment margin 
Table 1.4. DBAP 2007 Summary - Balance sheet by state and overall average, median, and standard deviation (\$/cow).

\begin{tabular}{|c|c|c|c|c|c|}
\hline \multirow[b]{2}{*}{ Category } & \multicolumn{3}{|c|}{ Overall } & \multicolumn{2}{|c|}{ State Averages } \\
\hline & Average & Median & $\operatorname{Std}^{1}$ & Florida & Georgia \\
\hline Number of farms & 21 & 21 & 21 & 16 & 5 \\
\hline \multicolumn{6}{|c|}{ Balance sheet (January 1): } \\
\hline Current assets & 378 & 305 & 300 & 309 & 599 \\
\hline Total assets & 6,821 & 5,597 & 4,575 & 5,807 & 10,064 \\
\hline Current liabilities & 638 & 502 & 557 & 511 & 1,044 \\
\hline Total liabilities & 1,704 & 1,509 & 1,090 & 1,448 & 2,525 \\
\hline Equity & 5,116 & 3,924 & 4,700 & 4,359 & 7,538 \\
\hline \multicolumn{6}{|c|}{ Balance sheet (December 31): } \\
\hline Current assets & 741 & 663 & 558 & 602 & 1,183 \\
\hline Total assets & 7,366 & 5,452 & 4,509 & 6,176 & 11,176 \\
\hline Current liabilities & 487 & 310 & 449 & 497 & 458 \\
\hline Total liabilities & 1,762 & 1,413 & 1,227 & 1,518 & 2,544 \\
\hline Equity & 5,604 & 4,149 & 4,710 & 4,658 & 8,631 \\
\hline
\end{tabular}

${ }^{1}$ Standard deviation 
Table 2.1. DBAP 2007 Summary - Business size and production efficiency by average number of cows and milk per cow.

\begin{tabular}{|c|c|c|c|c|c|c|}
\hline \multirow[b]{2}{*}{ Category } & \multicolumn{3}{|c|}{ Average number of cows } & \multicolumn{3}{|c|}{ Milk yield (Ibs / cow / year) } \\
\hline & $<600$ & $\begin{array}{l}600- \\
1800\end{array}$ & $>1800$ & $<16,100$ & $16,100-20,600$ & $>20,600$ \\
\hline Number of farms & 7 & 7 & 7 & 7 & 7 & 7 \\
\hline \multicolumn{7}{|l|}{ Business Size: } \\
\hline Average number of cows & 253 & 1,028 & 2,914 & 2,027 & 687 & 1,482 \\
\hline Average number of heifers & 150 & 757 & 1,217 & 609 & 432 & 1,084 \\
\hline Milk sold (million lbs) & 4.69 & 19.29 & 53.20 & 31.45 & 12.56 & 33.18 \\
\hline FTE workers & 6 & 18 & 43 & 26 & 12 & 30 \\
\hline Acres of pasture + cultivated land & 328 & 498 & 1,517 & 1,337 & 400 & 606 \\
\hline \multicolumn{7}{|l|}{ Production Efficiency: } \\
\hline Milk sold (Ibs / cow / year) & 17,647 & 19,258 & 18,325 & 14,757 & 18,515 & 21,957 \\
\hline Cows / FTE worker & 37 & 59 & 79 & 71 & 57 & 48 \\
\hline Milk sold (million lbs)/ FTE worker & 0.66 & 1.11 & 1.40 & 1.08 & 1.05 & 1.05 \\
\hline Cull rate & $33 \%$ & $36 \%$ & $38 \%$ & $35 \%$ & $37 \%$ & $34 \%$ \\
\hline
\end{tabular}


Table 2.2. DBAP 2007 Summary - Revenues and expenses by average number of cows and milk per cow (\$/cwt).

\begin{tabular}{|c|c|c|c|c|c|c|}
\hline \multirow[b]{2}{*}{ Category } & \multicolumn{3}{|c|}{ Average number of cows } & \multicolumn{3}{|c|}{ Milk yield (lbs / cow / year) } \\
\hline & $<600$ & $\begin{array}{l}600- \\
1800\end{array}$ & $>1800$ & $<16,100$ & $16,100-20,600$ & $>20,600$ \\
\hline Number of farms & 7 & 7 & 7 & 7 & 7 & 7 \\
\hline \multicolumn{7}{|l|}{ Revenues: } \\
\hline Milk sold & 22.15 & 22.50 & 23.13 & 23.15 & 22.83 & 21.79 \\
\hline Raised, leased cow sales & 1.84 & 0.97 & 0.74 & 1.87 & 0.68 & 1.00 \\
\hline Heifer sales & $(0.01)$ & 0.05 & 0.50 & $(0.58)$ & 0.43 & 0.69 \\
\hline $\begin{array}{l}\text { Gain on purchased livestock } \\
\text { Sales }\end{array}$ & $(0.24)$ & $(0.22)$ & $(0.35)$ & $(0.36)$ & $(0.21)$ & $(0.23)$ \\
\hline Other revenues & 0.63 & 1.11 & 1.21 & 0.12 & 1.01 & 1.82 \\
\hline Total revenues & 24.37 & 24.41 & 25.23 & 24.20 & 24.73 & 25.08 \\
\hline \multicolumn{7}{|l|}{ Expenses: } \\
\hline Personnel & 3.74 & 3.61 & 3.37 & 3.60 & 3.57 & 3.55 \\
\hline Purchased feed & 9.80 & 8.69 & 9.09 & 10.05 & 9.16 & 8.38 \\
\hline Crops & 0.72 & 0.75 & 0.30 & 0.55 & 0.73 & 0.50 \\
\hline Machinery & 1.08 & 1.55 & 0.75 & 1.09 & 1.34 & 0.96 \\
\hline Livestock & 1.66 & 1.68 & 1.51 & 1.62 & 1.69 & 1.53 \\
\hline Milk marketing & 1.14 & 1.01 & 1.26 & 1.14 & 0.99 & 1.27 \\
\hline Buildings and land & 0.32 & 0.84 & 0.65 & 0.44 & 0.48 & 0.89 \\
\hline Interest & 0.84 & 0.44 & 0.59 & 0.60 & 0.64 & 0.63 \\
\hline \multicolumn{7}{|l|}{ Depreciation: } \\
\hline Livestock & 0.83 & 0.63 & 1.06 & 0.90 & 1.12 & 0.50 \\
\hline Machinery & 0.55 & 0.54 & 0.27 & 0.48 & 0.39 & 0.49 \\
\hline Buildings & 0.34 & 0.45 & 0.30 & 0.46 & 0.31 & 0.32 \\
\hline Other expenses & 1.06 & 0.98 & 0.86 & 0.92 & 1.15 & 0.84 \\
\hline Total expenses & 22.08 & 21.18 & 20.01 & 21.84 & 21.56 & 19.87 \\
\hline Net farm income from operations & 2.29 & 3.23 & 5.22 & 2.36 & 3.17 & 5.21 \\
\hline Gain on sale of capital assets & $(0.01)$ & 0.21 & $(0.23)$ & $(0.23)$ & 0.21 & $(0.01)$ \\
\hline Net farm income & 2.28 & 3.43 & 4.98 & 2.13 & 3.37 & 5.20 \\
\hline
\end{tabular}


Table 2.3. DBAP 2007 Summary - Financial performance by average number of cows and milk per cow.

\begin{tabular}{|c|c|c|c|c|c|c|}
\hline \multirow[b]{2}{*}{ Category } & \multicolumn{3}{|c|}{ Average number of cows } & \multicolumn{3}{|c|}{ Milk yield (lbs / cow / year) } \\
\hline & $<600$ & $\begin{array}{l}600- \\
1800\end{array}$ & $>1800$ & $<16,100$ & $16,100-20,600$ & $>20,600$ \\
\hline Number of farms & 7 & 7 & 7 & 7 & 7 & 7 \\
\hline \multicolumn{7}{|l|}{ Liquidity: } \\
\hline Current ratio & 10.42 & 28.82 & 3.23 & 2.93 & 1.28 & 38.26 \\
\hline Working capital (\$) & $(2,854)$ & 11,103 & $1,099,484$ & 445,025 & $(45,488)$ & 708,196 \\
\hline \multicolumn{7}{|l|}{ Solvency: } \\
\hline Debt to asset ratio & 0.33 & 0.22 & 0.32 & 0.23 & 0.31 & 0.32 \\
\hline Equity to asset ratio & 0.67 & 0.78 & 0.68 & 0.77 & 0.69 & 0.68 \\
\hline Debt to equity ratio & 0.78 & 0.29 & 0.72 & 0.36 & 0.74 & 0.69 \\
\hline \multicolumn{7}{|l|}{ Profitability: } \\
\hline Rate of return on assets & 0.07 & 0.11 & 0.28 & 0.09 & 0.13 & 0.23 \\
\hline Rate of return on equity & 0.09 & 0.12 & 0.56 & 0.12 & 0.16 & 0.50 \\
\hline Operating profit margin ratio & 0.02 & 0.14 & 0.23 & 0.04 & 0.13 & 0.21 \\
\hline \multicolumn{7}{|l|}{ Financial efficiency: } \\
\hline Asset turnover rate & 0.67 & 0.77 & 1.14 & 0.62 & 0.90 & 1.07 \\
\hline Operating expense ratio & 0.81 & 0.78 & 0.70 & 0.81 & 0.77 & 0.71 \\
\hline Depreciation expense ratio & 0.07 & 0.07 & 0.07 & 0.08 & 0.07 & 0.05 \\
\hline Interest expense ratio & 0.03 & 0.02 & 0.02 & 0.03 & 0.03 & 0.03 \\
\hline NFIFO ratio ${ }^{1}$ & 0.08 & 0.13 & 0.21 & 0.09 & 0.13 & 0.21 \\
\hline \multicolumn{7}{|l|}{ Repayment capacity: } \\
\hline Cash flow coverage ratio & 16.38 & 37.24 & 6.93 & 6.95 & 3.40 & 50.20 \\
\hline Term debt coverage ratio $^{2}$ & 1.92 & 2.46 & 6.50 & 3.99 & 3.85 & 3.04 \\
\hline Capital replacement margin ${ }^{3}(\$)$ & 81,027 & 466,374 & $1,566,809$ & 794,787 & 443,402 & 876,021 \\
\hline
\end{tabular}

${ }^{1}$ Net farm income from operations ratio

${ }^{2}$ Term debt and capital lease coverage ratio

${ }^{3}$ Capital replacement and term debt repayment margin 
Table 2.4. DBAP 2007 Summary - Balance sheet by average number of cows and milk per cow $(\$ / \mathrm{cow})$.

\begin{tabular}{|c|c|c|c|c|c|c|}
\hline \multirow[b]{2}{*}{ Category } & \multicolumn{3}{|c|}{ Average number of cows } & \multicolumn{3}{|c|}{ Milk yield (lbs / cow / year) } \\
\hline & $<600$ & $\begin{array}{l}600- \\
1800\end{array}$ & $>1800$ & $<16,100$ & $16,100-20,600$ & $>20,600$ \\
\hline Number of farms & 7 & 7 & 7 & 7 & 7 & 7 \\
\hline \multicolumn{7}{|c|}{ Balance sheet (January 1): } \\
\hline Current assets & 335 & 278 & 520 & 380 & 278 & 476 \\
\hline Total assets & 9,152 & 6,577 & 4,733 & 7,164 & 5,665 & 7,633 \\
\hline Current liabilities & 664 & 605 & 645 & 528 & 683 & 704 \\
\hline Total liabilities & 2,156 & 1,643 & 1,314 & 1,631 & 1,799 & 1,684 \\
\hline Equity & 6,996 & 4,934 & 3,419 & 5,534 & 3,866 & 5,949 \\
\hline \multicolumn{7}{|c|}{ Balance sheet (December 31): } \\
\hline Current assets & 781 & 408 & 1,033 & 663 & 431 & 1,127 \\
\hline Total assets & 9,887 & 6,579 & 5,632 & 7,185 & 5,854 & 9,059 \\
\hline Current liabilities & 525 & 314 & 623 & 447 & 541 & 475 \\
\hline Total liabilities & 2,339 & 1,315 & 1,632 & 1,592 & 1,640 & 2,054 \\
\hline Equity & 7,548 & 5,264 & 4,000 & 5,593 & 4,215 & 7,005 \\
\hline
\end{tabular}


Table 3.1. DBAP 2007 Summary - Business size and production efficiency by net farm income per cwt and rate of return on assets.

\begin{tabular}{|c|c|c|c|c|c|c|}
\hline \multirow[b]{2}{*}{ Category } & \multicolumn{3}{|c|}{ Net farm income (\$ / cwt) } & \multicolumn{3}{|c|}{ Rate of return on assets (\%) } \\
\hline & $<\$ 2.80$ & $\begin{array}{l}\$ 2.80 \\
-\$ 5.50\end{array}$ & $>\$ 5.50$ & $<5.0 \%$ & $\begin{array}{r}5.0 \%- \\
18.0 \%\end{array}$ & $>18.0 \%$ \\
\hline Number of farms & 7 & 7 & 7 & 7 & 7 & 7 \\
\hline \multicolumn{7}{|l|}{ Business Size: } \\
\hline Average number of cows & 699 & 2,348 & 1,148 & 773 & 889 & 2,534 \\
\hline Average number of heifers & 451 & 1,164 & 509 & 350 & 721 & 1,053 \\
\hline Milk sold (million lbs) & 12.56 & 43.66 & 20.97 & 12.60 & 18.75 & 45.84 \\
\hline FTE workers & 14 & 36 & 17 & 12 & 19 & 36 \\
\hline Acres of pasture + cultivated land & 371 & 1,237 & 735 & 577 & 435 & 1,331 \\
\hline \multicolumn{7}{|l|}{ Production Efficiency: } \\
\hline Milk sold (lbs / cow / year) & 16,569 & 18,824 & 19,836 & 16,416 & 20,539 & 18,275 \\
\hline Cows / FTE worker & 46 & 70 & 60 & 51 & 48 & 77 \\
\hline Milk sold (million lbs)/ FTE worker & 0.77 & 1.24 & 1.16 & 0.82 & 0.98 & 1.37 \\
\hline Cull rate & $40 \%$ & $37 \%$ & $30 \%$ & $38 \%$ & $35 \%$ & $33 \%$ \\
\hline
\end{tabular}


Table 3.2. DBAP 2007 Summary - Revenues and expenses by net farm income per cwt and rate of return on assets (\$/cwt).

\begin{tabular}{|c|c|c|c|c|c|c|}
\hline \multirow[b]{2}{*}{ Category } & \multicolumn{3}{|c|}{ Net farm income (\$ / cwt) } & \multicolumn{3}{|c|}{ Rate of return on assets (\%) } \\
\hline & $<\$ 2.80$ & $\begin{array}{l}\$ 2.80 \\
-\$ 5.50\end{array}$ & $>\$ 5.50$ & $<5.0 \%$ & $\begin{array}{l}5.0 \%- \\
18.0 \%\end{array}$ & $>18.0 \%$ \\
\hline Number of farms & 7 & 7 & 7 & 7 & 7 & 7 \\
\hline \multicolumn{7}{|l|}{ Revenues: } \\
\hline Milk sold & 22.23 & 22.98 & 22.55 & 22.71 & 21.83 & 23.23 \\
\hline Raised, leased cow sales & 2.16 & 1.02 & 0.37 & 1.84 & 1.30 & 0.40 \\
\hline Heifer sales & $(0.41)$ & 0.34 & 0.61 & $(0.30)$ & 0.26 & 0.57 \\
\hline $\begin{array}{l}\text { Gain on purchased livestock } \\
\text { sales }\end{array}$ & $(0.53)$ & $(0.22)$ & $(0.05)$ & $(0.55)$ & $(0.08)$ & $(0.17)$ \\
\hline Other revenues & 0.05 & 1.14 & 1.76 & 0.44 & 1.33 & 1.19 \\
\hline Total revenues & 23.50 & 25.26 & 25.25 & 24.14 & 24.65 & 25.22 \\
\hline \multicolumn{7}{|l|}{ Expenses: } \\
\hline Personnel & 3.75 & 3.30 & 3.67 & 4.18 & 3.25 & 3.29 \\
\hline Purchased feed & 10.70 & 9.39 & 7.50 & 10.50 & 8.49 & 8.59 \\
\hline Crops & 0.49 & 0.62 & 0.67 & 0.52 & 0.56 & 0.70 \\
\hline Machinery & 1.27 & 0.94 & 1.18 & 0.99 & 1.21 & 1.18 \\
\hline Livestock & 1.57 & 1.63 & 1.64 & 1.91 & 1.59 & 1.35 \\
\hline Milk marketing & 0.98 & 1.19 & 1.24 & 1.02 & 1.36 & 1.03 \\
\hline Buildings and land & 0.66 & 0.53 & 0.62 & 0.56 & 0.66 & 0.58 \\
\hline Interest & 0.66 & 0.59 & 0.61 & 0.60 & 0.80 & 0.47 \\
\hline \multicolumn{7}{|l|}{ Depreciation: } \\
\hline Livestock & 0.90 & 0.97 & 0.66 & 0.85 & 0.70 & 0.98 \\
\hline Machinery & 0.60 & 0.38 & 0.38 & 0.52 & 0.54 & 0.30 \\
\hline Buildings & 0.46 & 0.35 & 0.27 & 0.48 & 0.38 & 0.23 \\
\hline Other expenses & 1.15 & 0.95 & 0.81 & 1.01 & 0.96 & 0.94 \\
\hline Total expenses & 23.20 & 20.83 & 19.24 & 23.14 & 20.50 & 19.64 \\
\hline Net farm income from operations & 0.30 & 4.43 & 6.01 & 1.00 & 4.15 & 5.58 \\
\hline Gain on sale of capital assets & $(0.00)$ & $(0.07)$ & 0.03 & $(0.01)$ & 0.21 & $(0.23)$ \\
\hline Net farm income & 0.30 & 4.36 & 6.04 & 0.99 & 4.36 & 5.35 \\
\hline
\end{tabular}


Table 3.3. DBAP 2007 Summary - Financial performance by net farm income per cwt and rate of return on assets.

\begin{tabular}{|c|c|c|c|c|c|c|}
\hline \multirow[b]{2}{*}{ Category } & \multicolumn{3}{|c|}{ Net farm income (\$ / cwt) } & \multicolumn{3}{|c|}{ Rate of return on assets (\%) } \\
\hline & $<\$ 2.80$ & $\begin{array}{l}\$ 2.80 \\
-\$ 5.50\end{array}$ & $>\$ 5.50$ & $<5.0 \%$ & $\begin{array}{c}5.0 \%- \\
18.0 \%\end{array}$ & $>18.0 \%$ \\
\hline Number of farms & 7 & 7 & 7 & 7 & 7 & 7 \\
\hline \multicolumn{7}{|l|}{ Liquidity: } \\
\hline Current ratio & 1.04 & 1.81 & 39.62 & 1.80 & 38.28 & 2.39 \\
\hline Working capital $(\$)$ & $(133,023)$ & $1,006,023$ & 234,733 & 52,792 & 379,274 & 675,667 \\
\hline \multicolumn{7}{|l|}{ Solvency: } \\
\hline Debt to asset ratio & 0.31 & 0.29 & 0.27 & 0.23 & 0.35 & 0.29 \\
\hline Equity to asset ratio & 0.69 & 0.71 & 0.73 & 0.77 & 0.65 & 0.71 \\
\hline Debt to equity ratio & 0.61 & 0.62 & 0.56 & 0.49 & 0.68 & 0.63 \\
\hline \multicolumn{7}{|l|}{ Profitability: } \\
\hline Rate of return on assets & 0.02 & 0.18 & 0.25 & 0.01 & 0.14 & 0.31 \\
\hline Rate of return on equity & 0.00 & 0.27 & 0.50 & $(0.01)$ & 0.20 & 0.59 \\
\hline Operating profit margin ratio & $(0.04)$ & 0.19 & 0.24 & $(0.03)$ & 0.18 & 0.23 \\
\hline \multicolumn{7}{|l|}{ Financial efficiency: } \\
\hline Asset turnover rate & 0.64 & 0.96 & 0.99 & 0.45 & 0.84 & 1.30 \\
\hline Operating expense ratio & 0.88 & 0.73 & 0.68 & 0.87 & 0.73 & 0.70 \\
\hline Depreciation expense ratio & 0.08 & 0.07 & 0.05 & 0.08 & 0.07 & 0.06 \\
\hline Interest expense ratio & 0.03 & 0.02 & 0.02 & 0.03 & 0.03 & 0.02 \\
\hline NFIFO ratio ${ }^{1}$ & 0.01 & 0.18 & 0.24 & 0.03 & 0.17 & 0.22 \\
\hline \multicolumn{7}{|l|}{ Repayment capacity: } \\
\hline Cash flow coverage ratio & 2.43 & 1.74 & 56.38 & 3.42 & 50.81 & 6.32 \\
\hline Term debt coverage ratio ${ }^{2}$ & 0.91 & 7.72 & 2.25 & 2.40 & 2.21 & 6.28 \\
\hline Capital replacement margin ${ }^{3}(\$)$ & 125,181 & $1,111,505$ & 877,524 & 282,830 & 207,067 & $1,624,313$ \\
\hline
\end{tabular}

${ }^{1}$ Net farm income from operations ratio

${ }^{2}$ Term debt and capital lease coverage ratio

${ }^{3}$ Capital replacement and term debt repayment margin 
Table 3.4. DBAP 2007 Summary - Balance sheet by net farm income per cwt and rate of return on assets ( $\$ /$ cow).

\begin{tabular}{|c|c|c|c|c|c|c|}
\hline \multirow[b]{2}{*}{ Category } & \multicolumn{3}{|c|}{ Net farm income (\$ / cwt) } & \multicolumn{3}{|c|}{ Rate of return on assets (\%) } \\
\hline & $<\$ 2.80$ & $\begin{array}{l}\$ 2.80 \\
-\$ 5.50\end{array}$ & $>\$ 5.50$ & $<5.0 \%$ & $\begin{array}{c}5.0 \%- \\
18.0 \%\end{array}$ & $>18.0 \%$ \\
\hline Number of farms & 7 & 7 & 7 & 7 & 7 & 7 \\
\hline \multicolumn{7}{|c|}{ Balance sheet (January 1): } \\
\hline Current assets & 242 & 565 & 326 & 260 & 484 & 390 \\
\hline Total assets & 7,442 & 5,222 & 7,798 & 10,734 & 6,140 & 3,588 \\
\hline Current liabilities & 444 & 1,020 & 451 & 322 & 1,037 & 556 \\
\hline Total liabilities & 1,981 & 1,704 & 1,428 & 1,719 & 2,343 & 1,052 \\
\hline Equity & 5,461 & 3,519 & 6,369 & 9,015 & 3,797 & 2,536 \\
\hline \multicolumn{7}{|c|}{ Balance sheet (December 31): } \\
\hline Current assets & 478 & 998 & 746 & 651 & 792 & 779 \\
\hline Total assets & 7,530 & 5,689 & 8,879 & 10,874 & 6,862 & 4,363 \\
\hline Current liabilities & 558 & 678 & 227 & 422 & 505 & 535 \\
\hline Total liabilities & 2,064 & 1,592 & 1,630 & 1,758 & 2,303 & 1,225 \\
\hline Equity & 5,465 & 4,097 & 7,250 & 9,116 & 4,559 & 3,138 \\
\hline
\end{tabular}


Table 4.1. DBAP 2007 Summary - Business size and production efficiency by assets per cow and liabilities per cow.

\begin{tabular}{|c|c|c|c|c|c|c|}
\hline \multirow[b]{2}{*}{ Category } & \multicolumn{3}{|c|}{ Assets $(\$ /$ cow $)$} & \multicolumn{3}{|c|}{ Liabilities ( $\$$ / cow) } \\
\hline & $<\$ 4,700$ & $\$ 4,700-\$ 8,000$ & $>\$ 8,000$ & $<\$ 1,200$ & $\$ 1,200-\$ 2,300$ & $>\$ 2,300$ \\
\hline Number of farms & 7 & 7 & 7 & 7 & 7 & 7 \\
\hline \multicolumn{7}{|l|}{ Business Size: } \\
\hline Average number of cows & 1,404 & 2,197 & 595 & 1,791 & 1,125 & 1,280 \\
\hline Average number of heifers & 692 & 1,192 & 241 & 950 & 736 & 439 \\
\hline Milk sold (million lbs) & 24.86 & 41.68 & 10.65 & 32.80 & 20.92 & 23.47 \\
\hline FTE workers & 25 & 33 & 9 & 32 & 18 & 17 \\
\hline $\begin{array}{l}\text { Acres of pasture + cultivated } \\
\text { land }\end{array}$ & 918 & 940 & 485 & 1,219 & 462 & 662 \\
\hline \multicolumn{7}{|l|}{ Production Efficiency: } \\
\hline Milk sold (Ibs / cow / year) & 18,394 & 18,680 & 18,156 & 18,720 & 17,501 & 19,009 \\
\hline Cows / FTE worker & 54 & 74 & 47 & 58 & 58 & 60 \\
\hline $\begin{array}{l}\text { Milk sold (million Ibs)/ FTE } \\
\text { worker }\end{array}$ & 1.00 & 1.32 & 0.85 & 1.04 & 1.05 & 1.08 \\
\hline Cull rate & $33 \%$ & $37 \%$ & $37 \%$ & $34 \%$ & $34 \%$ & $38 \%$ \\
\hline
\end{tabular}


Table 4.2. DBAP 2007 Summary - Revenues and expenses by assets per cow and liabilities per cow ( $\$ / c w t)$.

\begin{tabular}{|c|c|c|c|c|c|c|}
\hline \multirow[b]{2}{*}{ Category } & \multicolumn{3}{|c|}{ Assets ( $\$$ / cow) } & \multicolumn{3}{|c|}{ Liabilities (\$ / cow) } \\
\hline & $<\$ 4,700$ & $\$ 4,700-\$ 8,000$ & $>\$ 8,000$ & $<\$ 1,200$ & $\$ 1,200-\$ 2,300$ & $>\$ 2,300$ \\
\hline Number of farms & 7 & 7 & 7 & 7 & 7 & 7 \\
\hline \multicolumn{7}{|l|}{ Revenues: } \\
\hline Milk sold & 22.88 & 22.79 & 22.11 & 23.29 & 22.58 & 21.90 \\
\hline Raised, leased cow sales & 0.69 & 0.94 & 1.92 & 0.72 & 1.00 & 1.82 \\
\hline Heifer sales & 0.87 & $(0.01)$ & $(0.32)$ & 0.00 & 0.75 & $(0.21)$ \\
\hline $\begin{array}{l}\text { Gain on purchased livestock } \\
\text { Sales }\end{array}$ & $(0.14)$ & $(0.17)$ & $(0.50)$ & $(0.08)$ & $(0.37)$ & $(0.35)$ \\
\hline Other revenues & 1.12 & 1.28 & 0.54 & 1.66 & 0.29 & 1.00 \\
\hline Total revenues & 25.42 & 24.84 & 23.75 & 25.61 & 24.25 & 24.16 \\
\hline \multicolumn{7}{|l|}{ Expenses: } \\
\hline Personnel & 3.54 & 3.42 & 3.76 & 4.33 & 3.98 & 2.40 \\
\hline Purchased feed & 9.42 & 9.35 & 8.81 & 8.80 & 9.95 & 8.84 \\
\hline Crops & 0.64 & 0.51 & 0.63 & 0.78 & 0.52 & 0.48 \\
\hline Machinery & 1.15 & 1.18 & 1.05 & 0.99 & 1.37 & 1.03 \\
\hline Livestock & 1.14 & 1.81 & 1.89 & 1.93 & 1.57 & 1.35 \\
\hline Milk marketing & 1.05 & 1.18 & 1.18 & 1.05 & 1.04 & 1.32 \\
\hline Buildings and land & 0.74 & 0.54 & 0.52 & 0.61 & 0.86 & 0.33 \\
\hline Interest & 0.62 & 0.67 & 0.57 & 0.23 & 0.38 & 1.26 \\
\hline \multicolumn{7}{|l|}{ Depreciation: } \\
\hline Livestock & 1.22 & 0.61 & 0.69 & 0.51 & 0.76 & 1.26 \\
\hline Machinery & 0.38 & 0.39 & 0.60 & 0.26 & 0.52 & 0.58 \\
\hline Buildings & 0.19 & 0.46 & 0.44 & 0.21 & 0.53 & 0.34 \\
\hline Other expenses & 1.18 & 0.82 & 0.91 & 0.88 & 1.17 & 0.86 \\
\hline Total expenses & 21.28 & 20.95 & 21.05 & 20.58 & 22.65 & 20.04 \\
\hline Net farm income from operations & 4.14 & 3.89 & 2.70 & 5.03 & 1.59 & 4.12 \\
\hline Gain on sale of capital assets & $(0.23)$ & 0.21 & $(0.01)$ & $(0.03)$ & $(0.00)$ & $(0.00)$ \\
\hline Net farm income & 3.91 & 4.10 & 2.69 & 4.99 & 1.59 & 4.12 \\
\hline
\end{tabular}


Table 4.3. DBAP 2007 Summary - Financial performance by assets per cow and liabilities per cow.

\begin{tabular}{|c|c|c|c|c|c|c|}
\hline \multirow[b]{2}{*}{ Category } & \multicolumn{3}{|c|}{ Assets (\$ / cow) } & \multicolumn{3}{|c|}{ Liabilities ( $\$$ / cow) } \\
\hline & $<\$ 4,700$ & $\begin{array}{c}\$ 4,700-\$ 8 \\
000\end{array}$ & $>\$ 8,000$ & $<\$ 1,200$ & $\$ 1,200-\$ 2,300$ & $>\$ 2,300$ \\
\hline Number of farms & 7 & 7 & 7 & 7 & 7 & 7 \\
\hline \multicolumn{7}{|l|}{ Liquidity: } \\
\hline Current ratio & 2.09 & 1.23 & 39.15 & 3.21 & 0.93 & 38.33 \\
\hline Working capital (\$) & 287,239 & 549,984 & 270,509 & 913,547 & $(200,063)$ & 394,249 \\
\hline \multicolumn{7}{|l|}{ Solvency: } \\
\hline Debt to asset ratio & 0.40 & 0.29 & 0.18 & 0.12 & 0.29 & 0.46 \\
\hline Equity to asset ratio & 0.60 & 0.71 & 0.82 & 0.88 & 0.71 & 0.54 \\
\hline Debt to equity ratio & 1.08 & 0.47 & 0.24 & 0.15 & 0.60 & 1.04 \\
\hline \multicolumn{7}{|l|}{ Profitability: } \\
\hline Rate of return on assets & 0.26 & 0.15 & 0.04 & 0.18 & 0.15 & 0.13 \\
\hline Rate of return on equity & 0.53 & 0.21 & 0.04 & 0.21 & 0.38 & 0.19 \\
\hline Operating profit margin ratio & 0.17 & 0.18 & 0.03 & 0.19 & 0.02 & 0.18 \\
\hline \multicolumn{7}{|l|}{ Financial efficiency: } \\
\hline Asset turnover rate & 1.36 & 0.85 & 0.38 & 0.89 & 0.99 & 0.71 \\
\hline Operating expense ratio & 0.74 & 0.76 & 0.80 & 0.76 & 0.86 & 0.69 \\
\hline Depreciation expense ratio & 0.07 & 0.06 & 0.08 & 0.04 & 0.08 & 0.09 \\
\hline Interest expense ratio & 0.02 & 0.03 & 0.02 & 0.01 & 0.01 & 0.05 \\
\hline NFIFO ratio ${ }^{1}$ & 0.16 & 0.16 & 0.10 & 0.20 & 0.05 & 0.17 \\
\hline \multicolumn{7}{|l|}{ Repayment capacity: } \\
\hline Cash flow coverage ratio & 5.90 & 1.53 & 53.12 & 7.54 & 2.72 & 50.29 \\
\hline Term debt coverage ratio ${ }^{2}$ & 2.93 & 5.96 & 1.99 & 6.01 & 1.90 & 2.97 \\
\hline Capital replacement margin ${ }^{3}(\$)$ & 828,676 & $1,007,330$ & 278,204 & $1,197,609$ & 588,770 & 327,831 \\
\hline
\end{tabular}

${ }^{1}$ Net farm income from operations ratio

${ }^{2}$ Term debt and capital lease coverage ratio

${ }^{3}$ Capital replacement and term debt repayment margin 
Table 4.4. DBAP 2007 Summary - Balance sheet by assets per cow and liabilities per cow (\$/cow).

\begin{tabular}{|c|c|c|c|c|c|c|}
\hline \multirow[b]{2}{*}{ Category } & \multicolumn{3}{|c|}{ Assets (\$ / cow) } & \multicolumn{3}{|c|}{ Liabilities ( $\$$ / cow) } \\
\hline & $<\$ 4,700$ & $\$ 4,700-\$ 8,000$ & $>\$ 8,000$ & $<\$ 1,200$ & $\$ 1,200-\$ 2,300$ & $>\$ 2,300$ \\
\hline Number of farms & 7 & 7 & 7 & 7 & 7 & 7 \\
\hline \multicolumn{7}{|c|}{ Balance sheet (January 1): } \\
\hline Current assets & 270 & 535 & 328 & 343 & 148 & 643 \\
\hline Total assets & 3,220 & 5,249 & 11,993 & 7,423 & 6,303 & 6,736 \\
\hline Current liabilities & 543 & 878 & 494 & 392 & 415 & 1,107 \\
\hline Total liabilities & 1,302 & 1,866 & 1,945 & 753 & 1,356 & 3,004 \\
\hline Equity & 1,918 & 3,383 & 10,048 & 6,670 & 4,947 & 3,732 \\
\hline \multicolumn{7}{|c|}{ Balance sheet (December 31): } \\
\hline Current assets & 566 & 883 & 773 & 765 & 311 & 1,146 \\
\hline Total assets & 4,008 & 5,632 & 12,459 & 7,830 & 6,531 & 7,737 \\
\hline Current liabilities & 559 & 716 & 187 & 210 & 471 & 781 \\
\hline Total liabilities & 1,670 & 1,689 & 1,927 & 579 & 1,541 & 3,166 \\
\hline Equity & 2,338 & 3,943 & 10,532 & 7,251 & 4,990 & 4,571 \\
\hline
\end{tabular}

